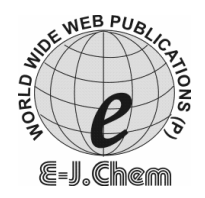

http://www.e-journals.net
ISSN: 0973-4945; CODEN ECJHAO

E-Journal of Chemistry 2010, 7(2), 403-408

\title{
Low-level Determination of 4-Hydrazino Benzoic Acid in Drug Substance by High Performance Liquid Chromatography/Mass Spectrometry
}

\author{
S. HARIKRISHNA*, K. NAGABHUSHANA REDDY, Y. SURESH, \\ M. RAMAKRISHNA, M. SARAT, K. KISHORE and C. RAMBABU \\ Analytical Development, \\ Matrix Laboratories Ltd, Hyderabad 500055, India. \\ harikrishnasontineni@yahoo.co.in
}

Received 6 August 2009; Accepted 2 October 2009

\begin{abstract}
HPLC/MS single ion monitoring methods have been evaluated for the determination of trace levels of 4- hydrazino benzoic acid (4-HBA) in drug substances. These 4-HBA have been highlighted as potential genotoxins. HPLC/MS was found to be more promising and limits of quantification was $0.52 \mathrm{ppm}$. For one drug substances excellent recoveries of $104.6 \%$ were obtained at the $0.5 \mathrm{ppm}$ level.
\end{abstract}

Keywords: HPLC, LC-HPLC/MS, LC-MS, Geotoxians, Trace analysis.

\section{Introduction}

4-Hydrazino benzoic acids are often used during manufacture of pharmaceuticals, either as counter-ions to form salt, as acid catalysts or as the result of protecting group removal during the synthesis. In fact 4-HBA is known genotoxins and is known carcinogens in rats and mice ${ }^{1}$. The potential presence of these genotoxins has attracted the attention of regulatory authorities, although no official guidelines have yet been issued. Draft guidelines $^{2}$ from the European agency and feedback from the US Food Drug Administration (FDA) to the pharmaceuticals industry via responses to drug applications have enabled the industry to establish interim strategies. Generally it is accepted that genotoxins will be limited to a daily dose of 1.0-1.5 $\mu \mathrm{g} /$ day unless safety studies establish that it is safe to receive a higher dose or that the drug is used for only a short term exposure, e.g. as an antibiotic. As some genotoxicity studies can take up to two years, e.g. 
carcinogenicity studies, it is preferable for the potential genotoxins to be controlled during the synthesis. In cases where levels cannot be controlled and no safety data yet exists it may be preferable for the pharmaceutical company to change the route of the drug substance or the isolation procedure, though this normally happens only during early development. This is a challenge analytically as well as synthetically as a $1.0 \mu \mathrm{g} /$ day dose transposes to $0.5 \mathrm{ppm}$ in the drug substances assuming a drug dose of $1 \mathrm{~g} /$ day.

Although the 4-HBA is well known carcinogens ${ }^{3-4}$, this data would infer that the regulatory authorities may be expected to request levels of 4-HBA to be controlled to 0.7 ppm in the drug substance (assuming a $1 \mathrm{~g}$ /day daily dose) or safety data to justify a greater dose. As matrix had one drug (API ), existing as a carboxylic acid salt, in the one stage development using 4-HBA as a catalyst in the synthesis, it was felt necessary to develop simple, sensitive and validated method for 4-HBA.

This paper ${ }^{5-6}$ describes a very brief evaluation of HPLC/MS approach, which has been used for the drug substances. As part of the approach, brief stability data were generated for the 4-HBA in various solvents as not all drug substances or intermediates is soluble in aqueous solutions.

\section{Experimental}

Samples of defrasirox and 4-HBA were received from the Process Research Department of Matrix Laboratories Limited, Hyderabad, India. HPLC grade acetonitrile and formic acid were purchased from Merck, Schuchardt, Germany. High pure water was prepared by using the Millipore Milli-Q Plus purification system.

\section{Preparation of solutions}

$0.5 \mathrm{mg} / \mathrm{mL}$ Concentrated stock solutions of the 4-HBA was prepared by dissolving the compounds in methanol. The diluted stock solution, $0.01 \mathrm{mg} / \mathrm{mL}$, was prepared by diluting $1 \mathrm{~mL}$ of the $0.5 \mathrm{mg} / \mathrm{mL}$ solutions to $50 \mathrm{~mL}$ with methanol.

\section{Instrumentation}

The LC-MS/MS system was used for method development and partial validation was a Applied Biosystems Sciex API 3000 model equipped with mass detector and auto sampler was used in the experiment. Data acquisition and processing were conducted using the Analyst software on a Pentium computer (Digital equipment Co).

\section{Operating conditions HPLC/MS/MS}

For the LC-MS/MS a Symmetry C18: $150 \mathrm{~mm} \times 4.6 \mathrm{~mm}, 5 \mu \mathrm{m}$ column (Waters Co,USA) in isocratic mode using $0.01 \mathrm{M}$ ammonium acetate and acetonitrile in the ratio $45: 55 \mathrm{v} / \mathrm{v}$. The flow rate was $1.0 \mathrm{~mL} / \mathrm{min}$, with the flow rate split down to $0.2 \mathrm{~mL} / \mathrm{min}$ in to the MS source. The column was monitored at $30{ }^{\circ} \mathrm{C}$ and the wavelength was set to 265 $\mathrm{nm}$ respectively. The injection volume was $30 \mu \mathrm{L}$. Electrospary in positive mode was used with or A selective ion monitoring (SIM) or selective ion-recording (SIR) mode was used as MS method for quantification of 4-HBA in drug substances. In this method 4-HBA is monitored by its molecular ion 153 (Protonated) and defrasirox was monitored by its molecular ion 374 (Protonated). The ion spray voltage (V), focusing potential, declustering potential and Entrance potential were 4500, 33,117 and 10 respectively. The curtain gas flow (psi) and nebulisation pressure (psi) are 6.0 and 4.0 respectively. 


\section{Results and Discussion}

The main target of the LC-MS/MS method was to separation and determination of 4-HBA impurity in the defrasirox active ingredient. Different reversed phase stationary phases have been assessed which included $\mathrm{C} 18, \mathrm{C} 8$ and cyano phases. In addition different mobile phases additives such as farmic acid, trifloro acetic acid perchloric acid, acetonitrile and methanol have been tested. Chromatographic separation was finally achieved on a Symmetry C18: $150 \mathrm{~mm} \times 4.6 \mathrm{~mm}, 5 \mu \mathrm{m}$ column (Waters Co, USA) in isocratic mode using $0.01 \mathrm{M}$ ammonium acetate and acetonitrile in the ratio 45:55 v/v. The flow rate was $1.0 \mathrm{~mL} / \mathrm{min}$, with the flow rate split down to $0.2 \mathrm{~mL} / \mathrm{min}$ in to the MS source.

\section{Validation of the method for 4-HBA in API}

Full validation data was required for API as it was in last stage development.

\section{Limit of detection (LOD) and limit of quantification (LOQ) for 4-HBA}

The LOD and LOQ were calculated from S/N data generated from six injections of 4-HBA (with out API) containing $0.52 \mathrm{ppm}$ of each 4-HBA with respect to an API sample concentration $10 \mathrm{mg} / \mathrm{mL}$. A LOQ of $0.52 \mathrm{ppm}$ is typical for the 4-HBA, with a LOD approximately three times less than LOQ. LOD and LOQ chromatograms are shown in the Figure $1 \& 2$

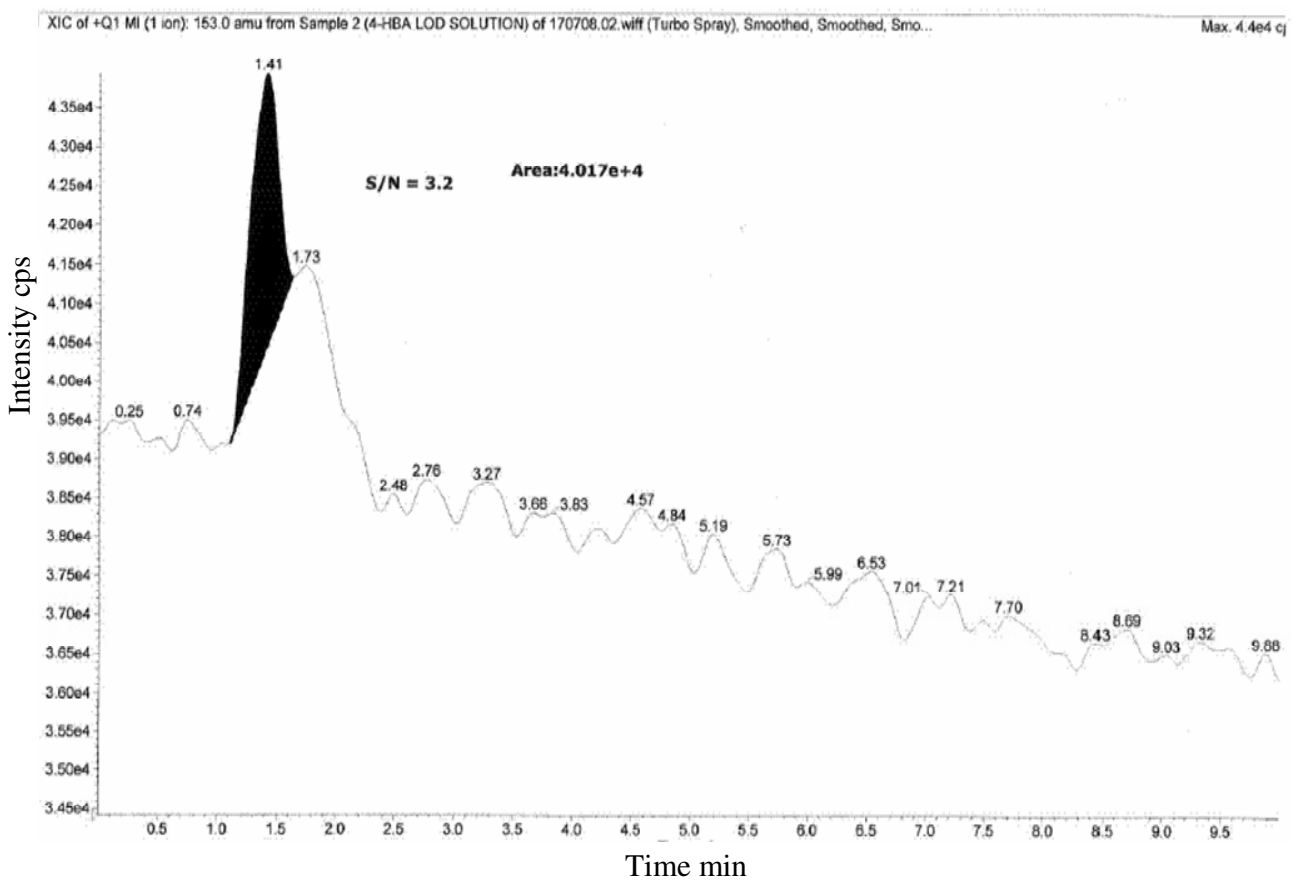

Figure 1. LOD chromatogram of 4-HBA.

\section{Recovery of 4-HBA from API}

The accuracy of the method was evaluated in triplicate at LOQ level in bulk drug sample. The percentage recoveries were calculated. A satisfactory recovery values of 4-HBA (98-111\%) was obtained. At such low levels these recoveries and \%RSD were satisfactory. Accuracy at LOQ chromatogram was shown in Figure 3. 


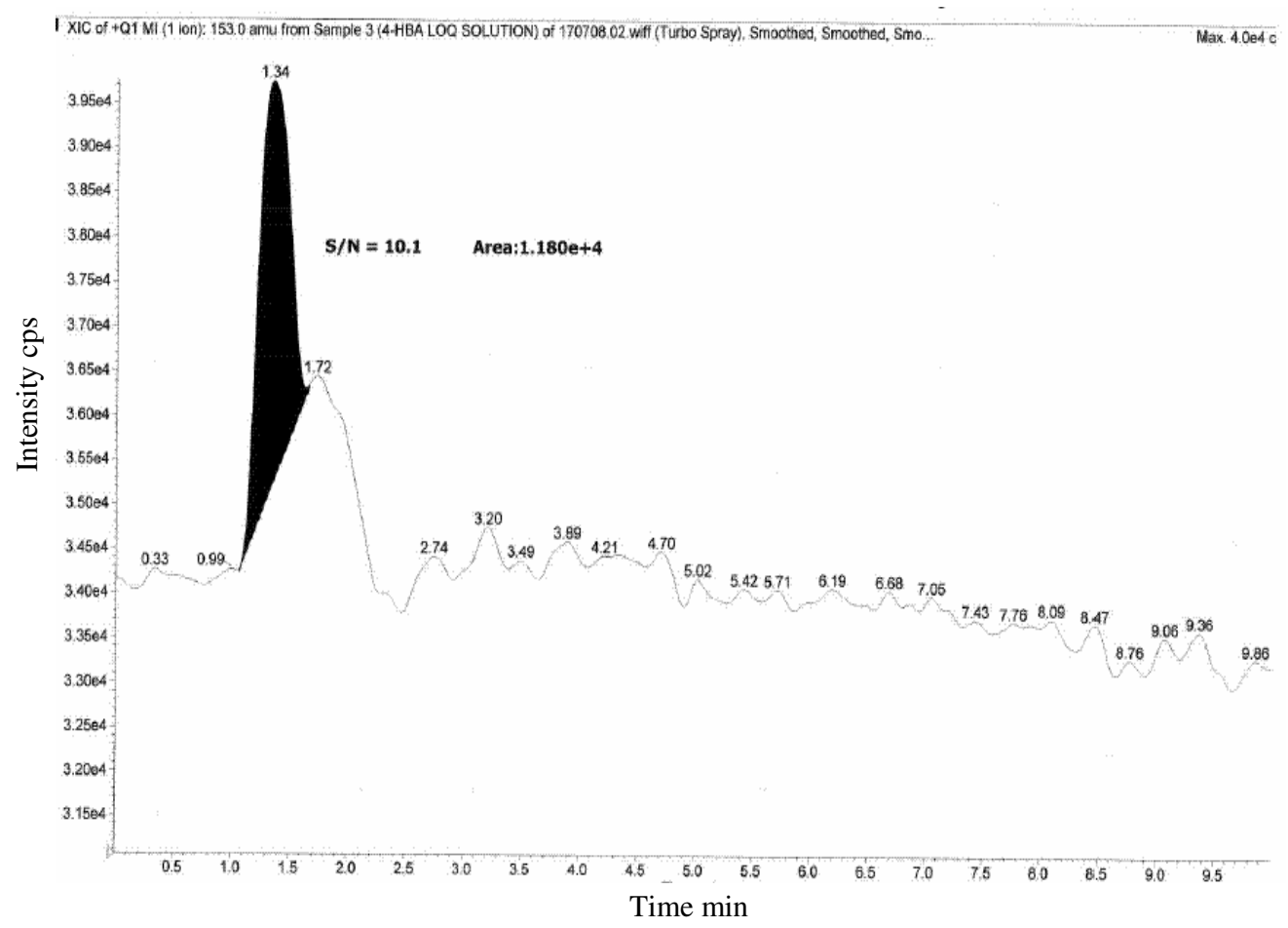

Figure 2. LOQ chromatogram of 4-HBA.

IXIC of +Q1 MI (1 ion): 153.0 amu from Sample 3 (DFR.VMK(A-078)51 Inj-1) of 170708.07. wift (Turto Spray). Smoothod, Smoothed, Sm... Max. 20046

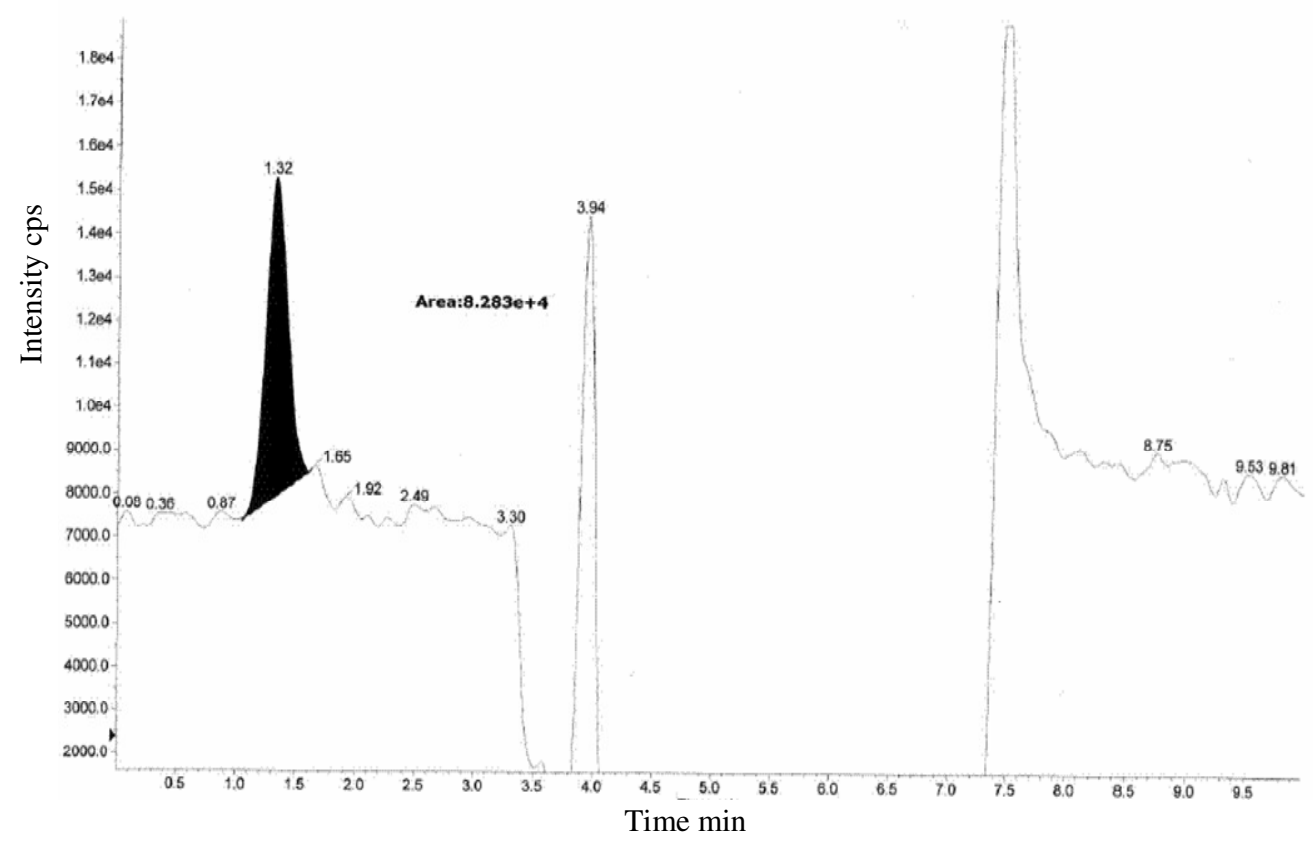

Figure 3. Accuracy at LOQ chromatograms of 4-HBA. 


\section{Linearity of the 4-HBA on the LC-MS/MS}

By selected ion monitoring, the linearity of 4-HBA was satisfactorily demonstrated with eight point calibration graph between LOQ to $150 \%$ of analyte concentrations (LOQ,25,50,100 and 150). The peak area versus concentration data was performed by least-squares linear regression analysis. The calibration curve was produced by plotting the average of triplicate 4-HBA injections against the concentration expressed in percentage. Correlation coefficients for 4-HBA was 0.99. Linearity of the 4-HBA chromatogram was shown in the Figure 4.

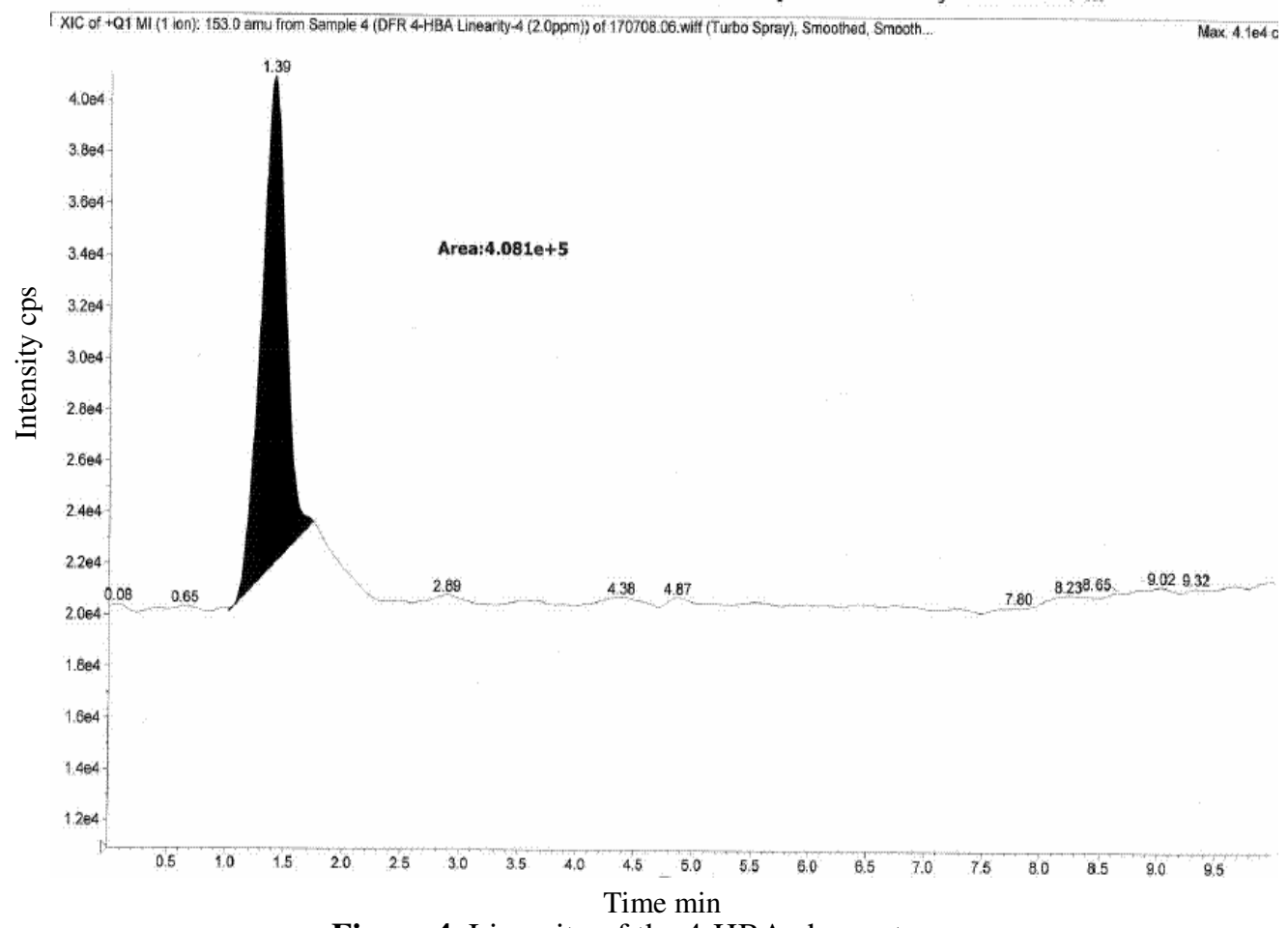

\section{Conclusions}

Figure 4. Linearity of the 4-HBA chromatogram.

LC-MS/MS using 4-HBA has been demonstrated to highly sensitive with limit of detection $0.17 \mathrm{ppm}$. Formic acid was used, it is important to add a trace level of ammonium acetate to the mobile phase to enhance ionization and detection. The methodology has one of the restrictions of 4-HBA of aqueous solution sample may be used; this approach is suitable for both API and intermediates. Sample solvents chosen should be assessed for the effect on standard stability with and without presence of API. Dramatic differences in stability were noted, therefore it is recommended recovery/ standard addition be carried to rule out stability or ion suppression issues.

\section{Acknowledgment}

The authors wish to thank the management of Matrix Laboratories Ltd., for supporting this work. Authors wish to acknowledge the Process Research Department for providing the samples for this research. We also would like to thank colleagues from the Separation Science Division of Analytical Development for their co-operation in carrying out this work. 


\section{References}

1. The European Medicines Agency [EMEA], Committee for Medical products for Human Use [CHMP], Guidelines on the Limits of Genotoxic Impurities CPMP/SWP/5199/02

2. Glowienke, Frieauff W, Allmendinger T, Martus H, Suter W and Mueller L, Mutat Res., 2005, 581, 23.

3 Colon I and Richoll S M, J Pharm Biomed Anal., 2005, 39, 477.

4 Ramjit H G, Singh M M and Coddington A B, J Mass Spectrom., 1996, 31(8), 867.

5. Lee C R, Guivarch F, Van Dau C N, Tessier D and Krstulovic A M, Analyst, 2003, 128, 857.

6. Li W, J Chromatogr A., 2004, 1046, 297. 


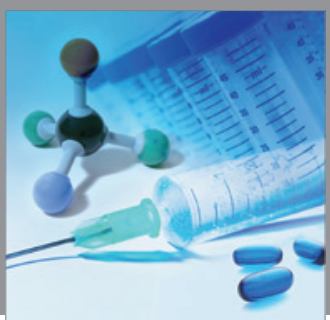

International Journal of

Medicinal Chemistry

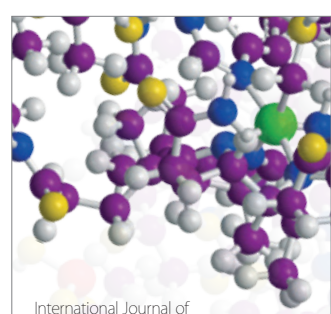

Carbohydrate Chemistry

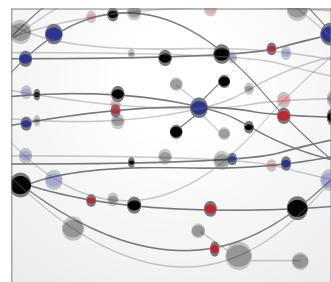

The Scientific World Journal
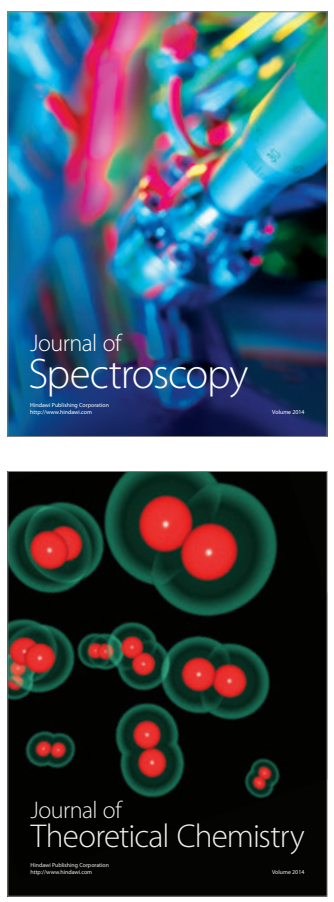
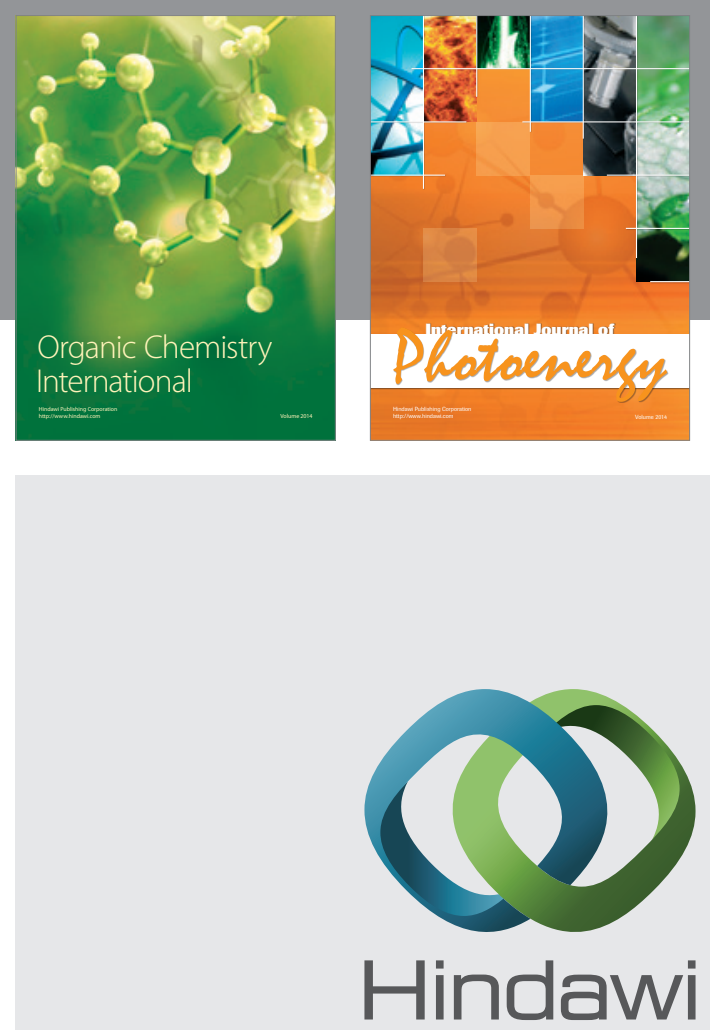

Submit your manuscripts at

http://www.hindawi.com
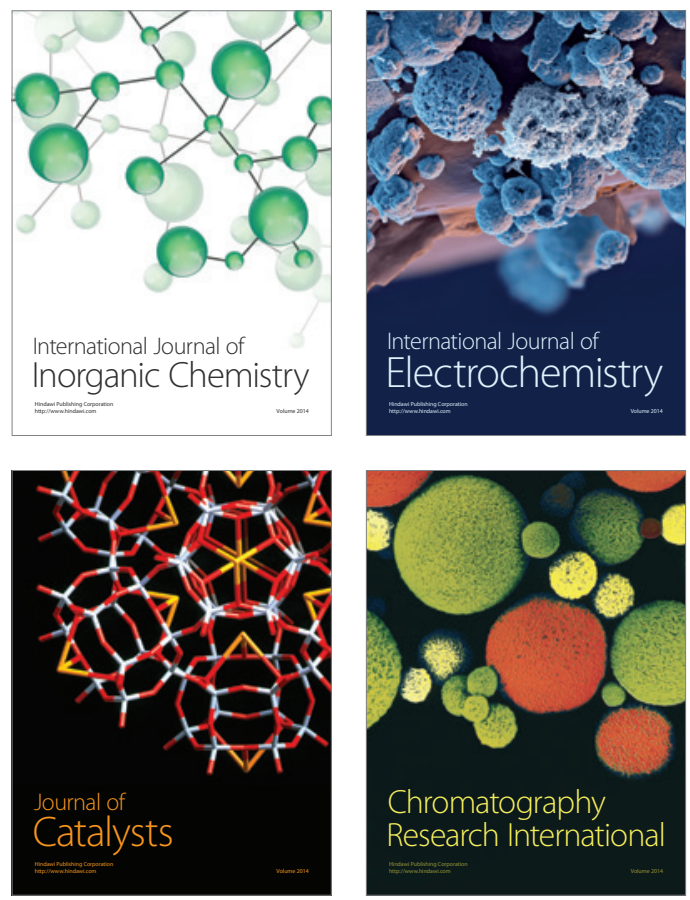
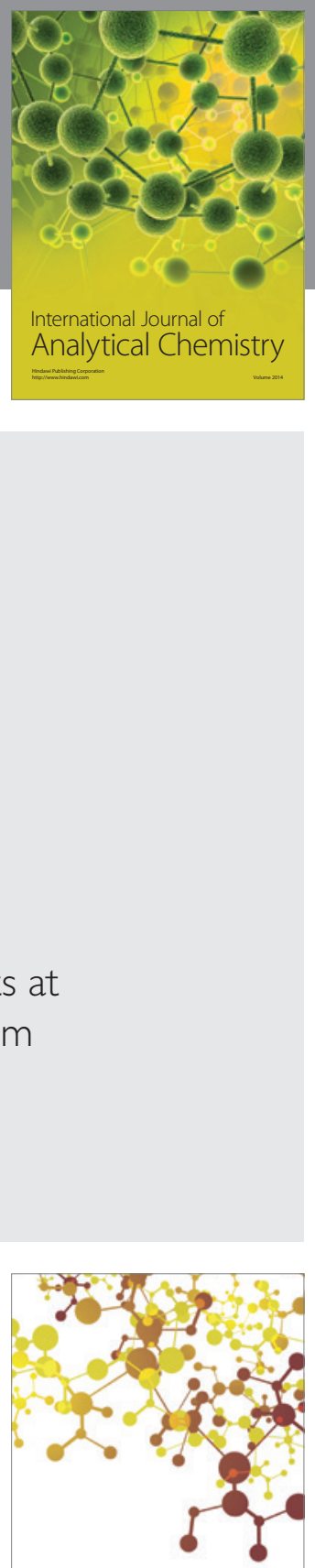

Journal of

Applied Chemistry
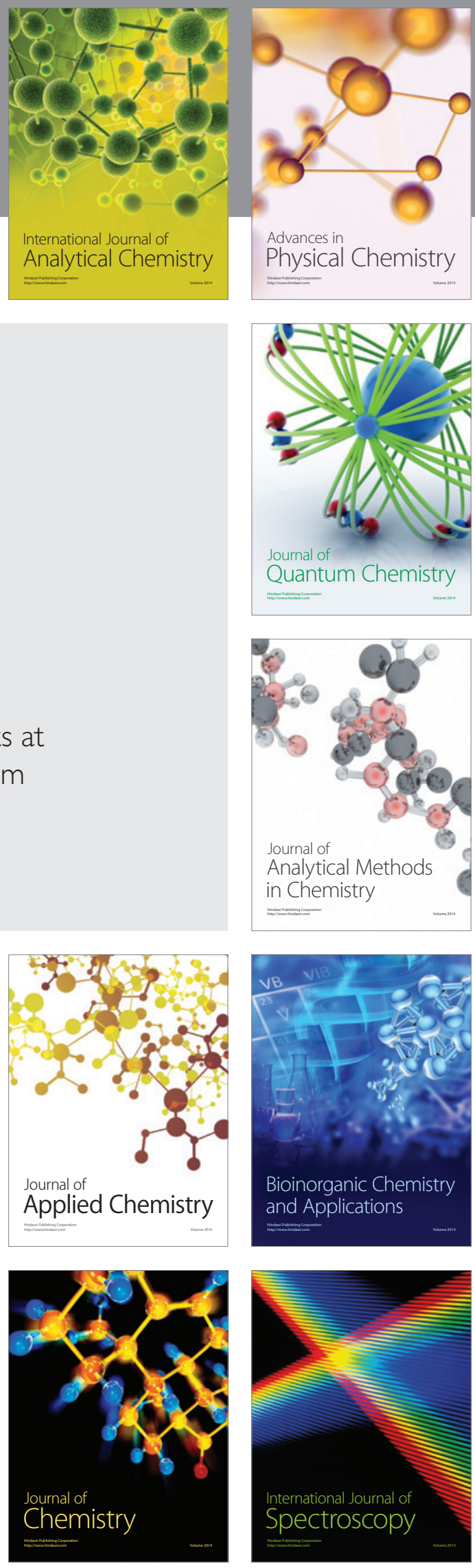